\title{
Pneumatic artificial muscles based on biomechanical characteristics of human muscles
}

doi:10.1533/abbi.2006.0028

\author{
N. Saga, J. Nagase and T. Saikawa \\ Department of Machine Intelligence and System Engineering, Akita Prefectural University, 015-0055 Akita, Japan
}

\begin{abstract}
This article reports the pneumatic artificial muscles based on biomechanical characteristics of human muscles. A wearable device and a rehabilitation robot that assist a human muscle should have characteristics similar to those of human muscle. In addition, since the wearable device and the rehabilitation robot should be light, an actuator with a high power to weight ratio is needed. At present, the McKibben type is widely used as an artificial muscle, but in fact its physical model is highly nonlinear. Therefore, an artificial muscle actuator has been developed in which high-strength carbon fibres have been built into the silicone tube. However, its contraction rate is smaller than the actual biological muscles. On the other hand, if an artificial muscle that contracts axially is installed in a robot as compactly as the robot hand, big installing space is required. Therefore, an artificial muscle with a high contraction rate and a tendon-driven system as a compact actuator were developed, respectively. In this study, we report on the basic structure and basic characteristics of two types of actuators.
\end{abstract}

Key words: Artificial muscle, tendon-driven system, isotonic contraction, isometric contraction, biomechanical characteristics.

\section{INTRODUCTION}

In recent times, our society has been aging rapidly, thus leading to a labour shortage of young workers. Therefore, robots are expected to be useful for works such as rehabilitation therapy, nursing elderly people, and day-to-day work support for elderly people. In particular, robots, which are intended for use in the field of medical care and welfare, should be safe for the human environment, as they often come into contact with people. The robots are required to have flexibility similar to human beings, and be able to perform like human movements.

In order to achieve this, the development of an artificial muscle actuator that is as soft as the muscle of a human being becomes indispensable. The main advantages of the artificial muscle are its high power to weight ratio, low price, low maintenance, and ability to be used in rough environments. Therefore, in the future, the artificial muscle can be used not only in the field of rehabilitation but also as an actuator in robots, including industrial robots, which are designed to cooperate with humans. At present,

\footnotetext{
Corresponding Author:

N. Saga

Department of Machine Intelligence and System Engineering

Akita Prefectural University, 015-0055 Akita, Japan

Tel: +81-184-27-2114; Fax: +81-184-27-2188

Email: saga@akita-pu.ac.jp
}

the McKibben type (Nickel et al. 1963; Gavrilovic and Maric 1969; Chou and Hannaford 1994; Klute et al. 1999) is widely used as an artificial muscle, but in fact its physical model is highly nonlinear (Caldwell et al. 1993; Chou et al. 1996; Repperger et al. 1999). Recently, a pneumatic muscle based on the McKibben type was produced by FESTO (FESTO, AG \& Co. 2001). Although this muscle has a high pulling force and a very long lifetime, it is also highly nonlinear. Therefore, it is necessary to use a complex controller with this artificial muscle in order to compensate for its nonlinear characteristics (Hildebrandt et al. 2002). The authors have developed an artificial muscle tube in which high-intensity Kevlar fibber has been built into a silicone tube (Saga et al. 2002, 2003). This actuator is longlasting, as it does not require a sleeve, and can express an aeolotropic property because of the way in which the fibber is knit into the tube. Furthermore, as many parameters regarding the shape of the tube are available, it is possible to flexibly design the artificial muscle tube. However, an actuator that takes the place of a human muscle is difficult to apply to a robot since the actuator's contraction rate is smaller than that of the actual biological muscles.

On the other hand, several robot hands similar to human hands have been developed. Some have a tendon-driven system and others have a direct system. In the former system (Liu et al. 1998), wire ropes in the finger parts are moved using an actuator with a DC motor incorporated into the back of the hand. In the latter system (Kawasaki 

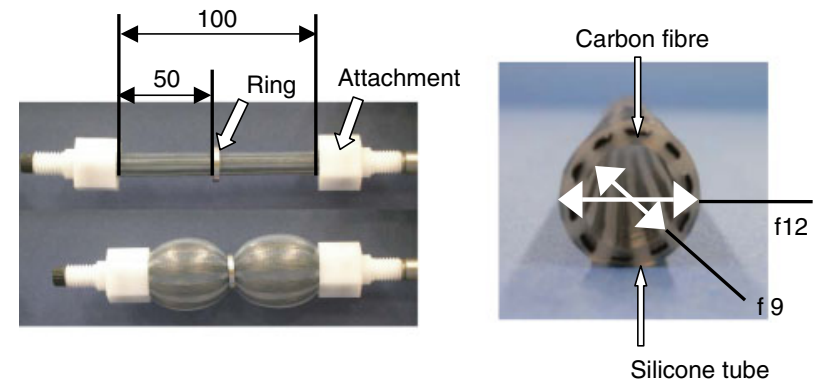

Figure 1 Photograph of the artificial muscle.

et al. 2001), an actuator is incorporated into the finger joints to enable a higher degree of joint freedom. However, they encounter some problems when compared to biological human hands. The former are bigger in size, and the strength of the driving power source is lower, whereas the latter are heavier and possess a complicated structure. Other robot hands were devised in order to realize flexible hands (Noritsugu et al. 2001; Yanagisawa et al. 2001): a robot hand with a rotary actuator made from silicon rubber in the joint, a hand with fingers made from rubber tube, and so on. However, they are very different from biological human hands about the joint freedom.

Therefore, we developed an artificial muscle actuator that has high shrinkage efficiency compared with the old type, as actuator of robot that is needed for comparatively big excursion. In addition, the tendon-driven system using a pneumatic balloon was developed as a soft actuator for the robot, with a small installing space for the actuator. This study reports on the basic structure and basic characteristics of these actuators. It also reports on the basic properties of the system evaluated as compared with the biological human muscle.

\section{DEVELOPED ARTIFICIAL MUSCLE ACTUATOR REINFORCED CARBON FIBERS IN THE AXIAL DIRECTION}

As shown in Figure 1, the constitution of the artificial muscle actuator is different and an improvement over the previous (McKibben) type. The tube for the actuator is composed of tubes based on a silicon rubber into which multiple carbon fibres are inserted vertically toward the direction of long axis. Therefore, a high contraction ratio can be achieved at low pressure, and the dilation is blocked axially. As a result, even when the tube is under internal pressure, the shrinkage characteristics similar to the muscle appear at the carbon fibres restrained at both ends. Moreover, the friction such as that in the McKibben type does not occur by the expansion and contraction of the sleeve type, and the heat and mechanical losses of this actuator are very small.

\section{Experimental setup}

Figure 2 shows the experimental setup using the artificial muscle. The artificial muscle is horizontally driven along

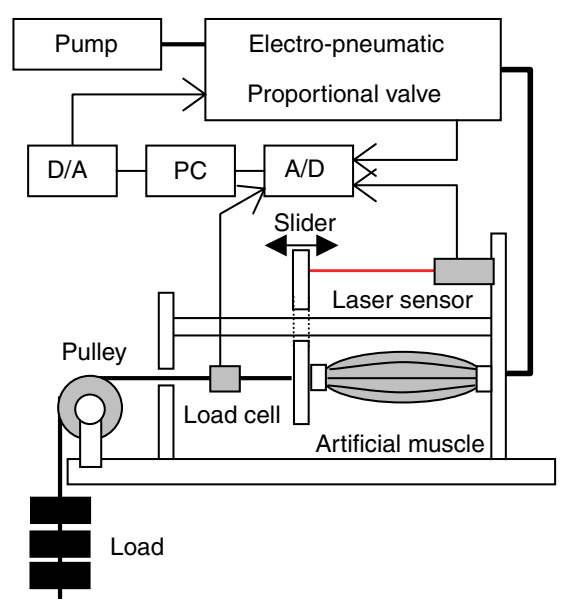

Figure 2 Configuration of the experimental setup.

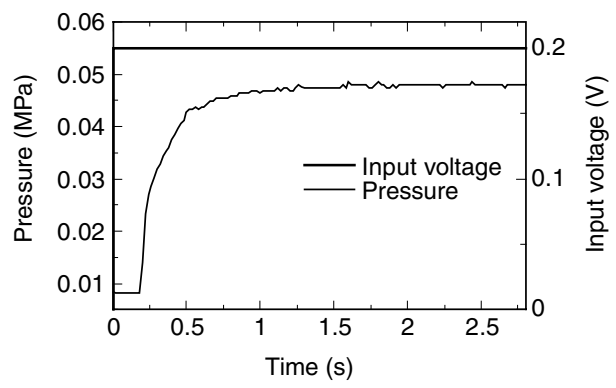

Figure 3 Step response of the electropneumatic proportional valve used by experimental setup.

the slider. Furthermore, the load can be applied in a perpendicular direction through the pulley. The contraction of the artificial muscle is controlled by an electro-pneumatic proportional valve (ETR200 KOGANEI), and is measured using a laser displacement sensor (LK-080 KEYENCE). The internal pressure of the artificial muscle is measured by means of a pressure sensor, which is positioned at the electro-pneumatic proportional valve. All of the measurement signals are recorded in a PC (DAQCard-6062E, National Instruments, Japan).

The step response characteristic of the valve is shown in Figure 3.

\section{Fundamental characteristics}

In this study, the characteristics of the proposed artificial muscle are discussed mainly in terms of its contraction behaviour. The artificial muscle is experimentally evaluated after testing its function several times.

Figure 4 shows the relationship between the quantity of inside pressure and the contraction of the artificial muscle. The initial length of the artificial muscle is $100 \mathrm{~mm}$. In the absence of a ring, the maximum contraction is $17 \mathrm{~mm}$, and with one ring it is $23 \mathrm{~mm}$, an increase of about 1.35 times. However, if we mount more than two rings, the amount of contraction decreases as compared to a single ring. From this, it is clear that mounting one ring can increase the contraction capability. An artificial muscle with one ring does 


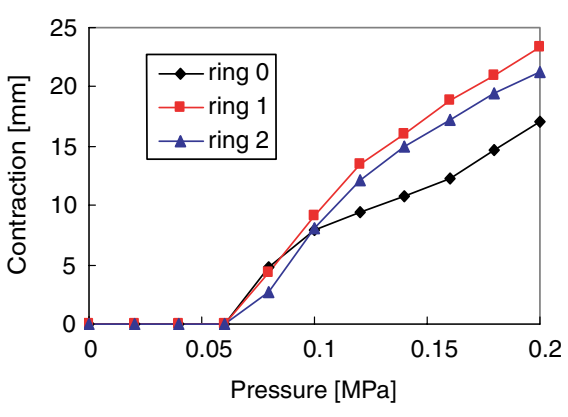

Figure 4 The relationship between pressure and contraction rate.

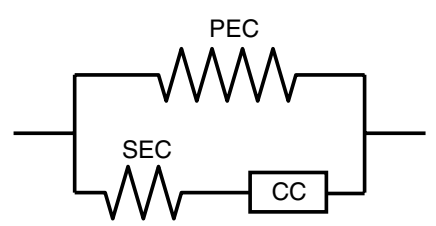

(a)

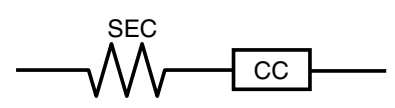

(b)

Figure 5 Schematic of a biomechanical muscle modelling experimentally established by Hill: (a) three-element muscle model; (b) two-element muscle model.

not expand to the vicinity of $0.08 \mathrm{MPa}$, and no contraction occurs. With an inside pressure of more than $0.16 \mathrm{MPa}$, the ratio of amount of contraction decreases and asymmetry can be seen. This means that because of the structure of artificial muscle, expansion is restricted due to the inserted fibber at an inside pressure of more than $0.16 \mathrm{MPa}$. The maximum contraction of $23 \mathrm{~mm}$ can be obtained at an inside pressure of $0.2 \mathrm{MPa}$.

\section{On the biomechanical muscle model}

A biomechanical muscle modelling has been experimentally established by Hill (Hill 1938). Figure 5(a) shows schematic of a three-element muscle model proposed by Hill. As shown in this figure, the model is composed of a contractile component (CC), a series elastic component (SEC), and a parallel elastic component (PEC). The results of this model are often used to predict force, length, and velocity relationships describing muscle behaviour. Here, the PEC is a nonlinear elastic element that does not follow Hook's law. It generates tension at more than a resting length. However, the PEC is often ignored in skeletal muscle models since the force across the element is insignificant until the muscle is stretched beyond its physiological range. Therefore, in this study, we used a two-element model as shown in Figure 5(b) as the artificial muscle model.

In order to investigate the biomechanical characteristics of this artificial muscle, the muscle's isotonic and isometric characteristics are discussed on the basis of the skeletal model, as shown in Figure 3. In general, these methods are used to investigate the characteristics of biological muscle only. In this study, by applying the characteristics of the artificial muscle a comparison of the biological muscle with the artificial muscle has been done.

\section{Isotonic contraction and Hill's muscle model}

First of all, isotonic contraction is discussed. The isotonic contraction indicates motions of contraction when the tension of the muscle balances a constant load. Under isotonic conditions, the output force of the muscle is a function of both length and velocity for a given level of activation. It is well known that the output force of biological muscle drops significantly as the contraction velocities increase during shortening. The general equation of this relationship is given as the Hill muscle model as follows:

$$
(F+a)(V+b)=P,
$$

where $F$ and $V$ are the muscle force and the contraction velocity, respectively; $a, b$ and $P$ (the power generated by the muscle) are constants. Here, in the case of $F=0$, the contraction velocity has the maximum value, and is shown in the following equation:

$$
V_{\mathrm{m}}=\frac{P-a b}{a},
$$

where $V_{\mathrm{m}}$ is the maximum contraction velocity. Similarly, when $V=0$, the muscle force has the maximum value, $F_{\mathrm{m}}$, as is given in Equation (3),

$$
F_{\mathrm{m}}=\frac{V-a b}{b} .
$$

When Equation (1) is divided by $V_{\mathrm{m}}$ and $F_{\mathrm{m}}$, Equation (4)

$$
\left(\frac{F}{F_{\mathrm{m}}}+C\right)\left(\frac{V}{V_{\mathrm{m}}}+C\right)=C(C+1)
$$

is obtained, where $C$ is a constant. Furthermore, the constant $C$ does not change with the temperature, although constants $a, b$ and $P$ are dependent on the temperature. The constant $C$ can be derived as follows:

$$
C=\frac{a}{F_{\mathrm{m}}}=\frac{b}{V_{\mathrm{m}}}=\frac{a b}{P-a b} .
$$

In the case of an actual human muscle, the range of values for $C$ is $0.12 \leq \mathrm{C} \leq 0.41$ (Wilkie 1950), and its average is 0.25 . Figure 6 shows the relationship between force and contraction velocity of artificial muscles on the basis of Equation (4). Furthermore, the characteristic of the human muscle (for $C=0.25$ ) is shown in this figure for reference. It shows that the characteristics of the proposed artificial muscle are qualitatively equal to those of a human muscle. Especially, when applying a pressure of $0.1 \mathrm{MPa}$, we can see that the behaviour of the artificial muscle is the closest to that of an actual human muscle. 


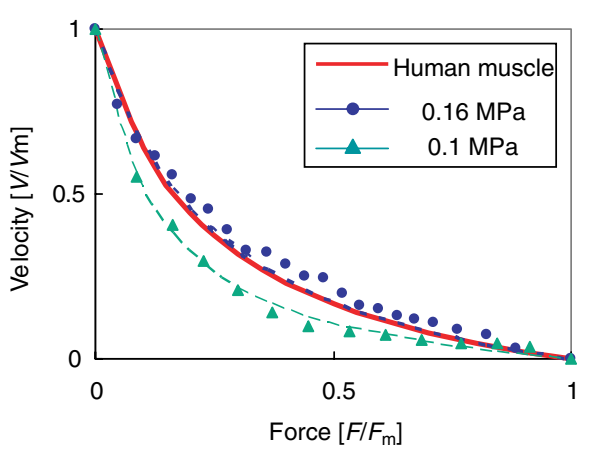

Figure 6 The relationship between force and contraction velocity based on the Hill muscle model.

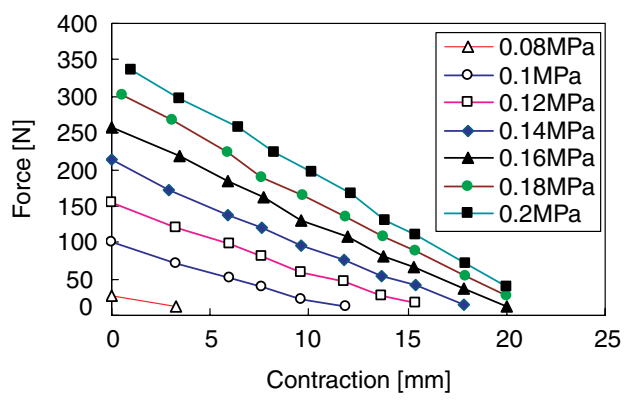

Figure 7 The relationship between force and contraction.

\section{Isometric contraction}

Next, the relationship between force and length during isometric contraction of the artificial muscle is discussed. Isometric contraction implies the contraction characteristics of the muscle when the muscle is fixed at a constant length. Therefore, the length of the muscle is maintained at a constant value by fixing the slide as in Figure 2, and muscle force is measured at each constant length. Figure 7 shows the relationship between the contraction ratio and contraction power of the artificial muscle under the above condition. The test conditions restrict the amount of contraction of an artificial muscle at random, and measured the contraction power of each at $0.08,0.1,0.12,0.14,0.16$, 0.18 , and $0.2 \mathrm{MPa}$ of the inside pressure by a road cell. The maximum contraction power was found to be more than $300 \mathrm{~N}$. As for the relationship between the contraction ratio and amount of contraction, contraction power increases as pressure increases. In addition, an artificial muscle shows the maximum contraction power with the natural length, and contraction power decreases as the contraction ratio increases. This means that when using an artificial arm actuator for robot arms, the initial torque can be effectively utilized.

\section{TENDON-DRIVEN SYSTEM USING A PNEUMATIC BALLOON}

\section{Prototype of a tendon-driven system}

The developed tendon-driven system consists mainly of a flat silicon tube and a tendon part. The specifications of the

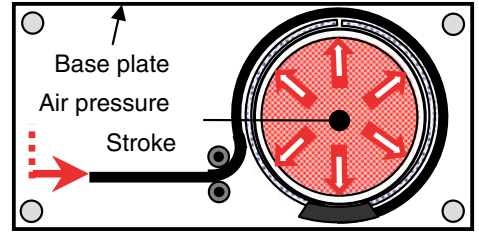

Figure 8 Mechanism of a tendon-driven system.

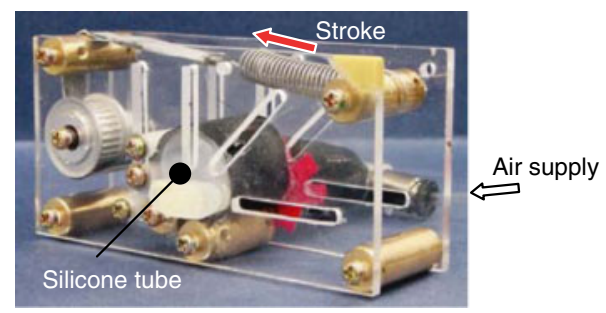

Figure 9 Prototype of a tendon-driven system.

Table 1 Specification of the silicone tube ${ }^{\mathrm{a}}$

\begin{tabular}{lll}
\hline & $\begin{array}{l}\text { Long diameter } \\
(\mathrm{mm})\end{array}$ & $\begin{array}{l}\text { Short diameter } \\
(\mathrm{mm})\end{array}$ \\
\hline Outer diameter $(\mathrm{mm})$ & 18.5 & 7.5 \\
Inner diameter $(\mathrm{mm})$ & 15.5 & 4.3 \\
\hline
\end{tabular}

${ }^{\text {a }}$ Dimensions of the silicone tube used: material, silicone rubber; effective length, $25 \mathrm{~mm}$; and weight, $5 \mathrm{~g}$.

tube are shown in Table 1 . When selecting the material for the tube, silicone rubber for medical use and natural rubber were examined and compared to each other. Silicon rubber was chosen because it had less residual strain compared to the natural rubber. It was considered that silicon rubber would show high response reproducibility when it is used as an actuator because it has strong linearity. The dimensions of the tube are as follows: the long outside diameter is $18.5 \mathrm{~mm}$ and the short outside diameter is $7.5 \mathrm{~mm}$, whereas the long inside diameter is $15.5 \mathrm{~mm}$ and the short inside diameter is $4.3 \mathrm{~mm}$. The effective length is $25 \mathrm{~mm}$, which is the upper limit.

The basic structure of the tendon-driven system is shown in Figure 8. The driving state of the actuator, when the tip of the sheet is attached to a spring with one end fixed, is shown in Figure 9. One end of the tube is closed to make it balloon-shaped, and the strip of non-elastic fibre is arranged around the tube. Air enters from the other end of the tube. The tube expands as it fills with air, and the nonelastic fibre around the tube generates a tensile strength, which provides our tendon-driven system. In this study, a strip-shaped non-elastic nylon sheet with a high-tensile strength was used as a tendon in order to create the strength of the driving power source.

\section{Basic characteristics}

In order to investigate the basic characteristics of the tendon-driven system prototype, experiments on the stroke characteristics against pressure and step response 


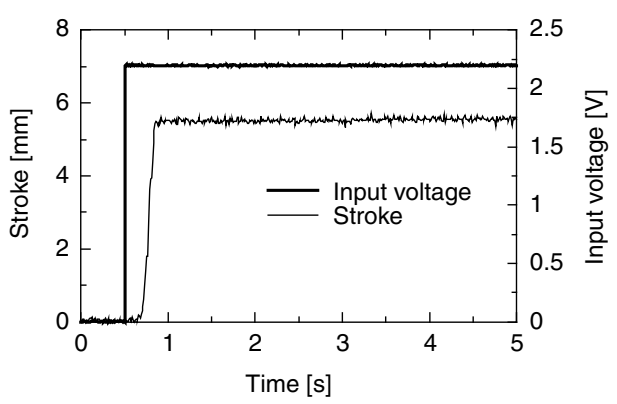

Figure 10 Step response of the proposed tendon-driven system.

characteristics were done. The experimental setup is the same as in Figure 2. In the experiment, the number of strokes by the tendon-driven system was measured using a laser sensor, and the generative force was measured with a load cell; and the measurement results were collected in a data logger. Also, a limiter was provided to control the tube displacement to within $25 \mathrm{~mm}$, because the tube might continue to expand until it bursts if pressure is applied continuously. Air pressure was applied to the actuator in increments of $0.05 \mathrm{MPa}$ until a limit of $25 \mathrm{~mm}$ was reached, and the maximum stroke at each input pressure was measured. Moreover, changes in the stroke characteristics under different loads, when the same air pressure mentioned above was applied, were also examined to compare the characteristics without a load. The load was 250 , 500 , and $750 \mathrm{~g}$ of weight, respectively, which was attached to one end of the actuator by a pulley.

Figure 10 shows the response characteristics of the actuator when pressure is applied in incremental steps. The input pressure is $0.04 \mathrm{MPa}$. The time constant is $0.29 \mathrm{~s}$, rise time is $0.11 \mathrm{~s}$, and the dead time is $0.17 \mathrm{~s}$, which indicates that the actuator has transient response characteristics. This is favourable because the volume of the actuator is small and it is less affected by flow characteristics, compared to larger pneumatic actuators.

\section{Biomechanical characteristics}

Furthermore, we examined the actuator to see whether it had biomechanical characteristics. Experiments on isotonic and isomeric contractions, which are ordinarily used for the assessment of human muscles, were done on the actuator to compare them with living body muscles. The aim was to see whether the same movement as the contraction of human muscles could be executed by the tendon-driven system when the system is installed in a robot hand and is allowed to grasp an object. Isotonic contraction generally refers to muscle contraction under a certain load, corresponding with the ordinary bending response of human fingers, whereas isometric contraction refers to the still force that muscles generate, that is to say, muscle contraction without changes in the length, corresponding with the pressure control function in grasping an object without changes in bending angles of human fingers.

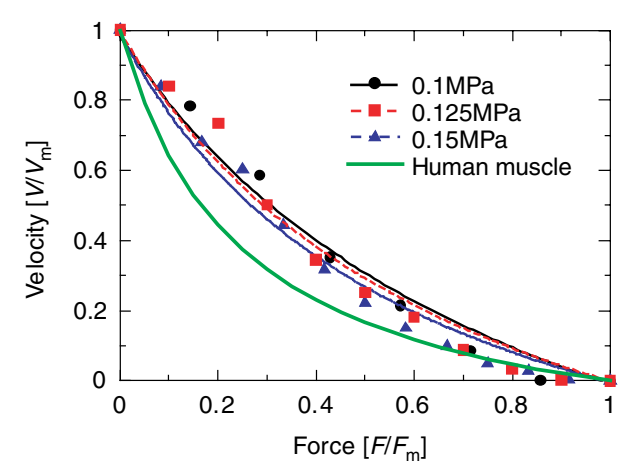

Figure 11 The relationship between force and velocity.

\section{Isotonic contraction}

In this study, the evaluation of the isotonic contraction was done as follows:

A certain load was applied to the actuator at its natural length through the pulley in incremental steps, with one of its end that was not fixed. The strokes at each pressure were measured using a laser sensor to examine the relationship between the load and stroke velocity. A stopper was set so that the stroke would not stretch beyond the natural length.

The variation in contraction velocity as the load was increased was examined, and the characteristics of the actuator and those of living body muscles under each pressure were compared to evaluate the actuator. The input pressure was $0.1,0.125$ and $0.15 \mathrm{MPa}$, respectively, and the load was applied in increments of $100 \mathrm{~g}$ until a weight at which the stroke was beyond the measurement length was reached.

Figure 11 shows the stroke velocity curves against the load, based on Equation (4). As the generated force and the loads were different under each pressure, each value was rendered as dimensionless numbers and presented in a graphical form. The heavy line in the figure represents the characteristics of a human muscle. The figure shows that the stroke velocity using load as the variable decreased and exhibited a hyperbolic curve as the load was increased, which is the same as the characteristics of human muscles. The dynamic traits of the actuator in this study are similar to human muscles, which indicate that flexible movements by human muscle contraction would be possible with this actuator. It was also found that the characteristics of the actuator became closer to human muscles as the input pressure was increased. The curve is exhibited only by the biggest contraction of human muscles, and the characteristics of the tendon-driven actuator in this study might be closer to human muscles by applying pressure that will generate a similar force of contraction.

\section{Isometric contraction}

An experiment to evaluate the isometric contraction of the actuator was performed, and the results were compared with living body muscles. The strokes of the actuator were limited at a certain degree and pressure was applied. Then, 


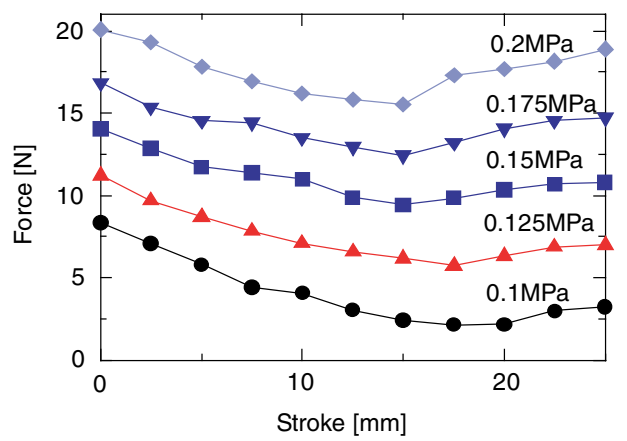

Figure 12 The relationship between stroke and force.

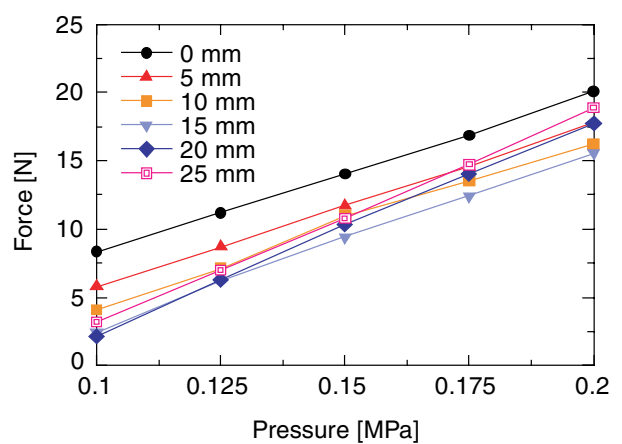

Figure 13 The relationship between pressure and force.

the generated force was measured to examine its relationship with the stroke. How the contraction length affected the generated force was also examined. Moreover, the variation of generative power as the pressure was increased was also considered.

The experimental method is as follows: The stroke was between $0 \mathrm{~mm}$ and $25 \mathrm{~mm}$, and was increased in increments of $2.5 \mathrm{~mm}$. The displacement of the stroke was fixed with a stopper at each length limit, to which pressure was applied in incremental steps, and the generated force was measured with a load cell. The pressure was between 0.10 and $0.20 \mathrm{MPa}$, and was applied in increments of $0.025 \mathrm{MPa}$.

The experimental results are shown in Figure 12. The generated force decreases at all pressure values when the stroke becomes shorter than $15 \mathrm{~mm}$. But the force increases as the stroke becomes longer than about 15 to $17.5 \mathrm{~mm}$ or more. This is different from the isometric contraction of living body muscle, where generated force decreases along with the muscle contraction, thus exhibiting a quadratic curve. Figure 13 shows the proportionality between air pressure and generated power, regardless of the value of strokes. This indicates that the grasping power of robot hands can be easily controlled without varying the angles of fingers if the relationship between the driving pressure and grasp pressure is mapped in advance.

\section{CONCLUSION}

In this article, an artificial muscle with a higher contraction rate and a tendon-driven system were developed.
Furthermore, the fundamental and biomechanical characteristics of these actuators were discussed. The conclusions are as follows:

- It is clear that the maximum contraction rate of developed artificial muscle is $20 \%$.

- For example, when applied to the robot hand, the developed tendon-driven system does not need such a large space as the conventional McKibben artificial muscle actuator, which use the contraction in the direction of the hand of the length, because the tendon can be arranged in the finger and the silicone tube is arranged in the relatively spacious palm. We feel that the size, weight and mechanical flexibility of this actuator are comparable to those of the human hand and that a safe design for the human environment is possible.

- In order to investigate the biomechanical characteristics of developed actuators, the isotonic and isometric characteristics of the muscle were discussed. Experimental results demonstrated the effectiveness of the artificial muscle including its biological characteristics, because the behaviour of the artificial muscle expresses the same tendencies as the human muscle.

\section{REFERENCES}

Caldwell DG, Razak A, Goodwin M. 1993. Braided pneumatic muscle actuators. In Proceedings of the IFAC Workshop on Intelligent Autonomous Vehicles, Sapporo, Japan, p. 507-12.

Chou CP, Hannaford B. 1994. Static and dynamic characteristics of MaKibben pneumatic artificial muscles. In Proceedings of IEEE International Conference On Robotics and Automation, San Diego, CA, p. 281-286.

Chou CP, Hannaford B. 1996. Measurement and modeling of McKibben pneumatic artificial muscles. IEEE Transaction on Robotics and Automation, 12-1, p. 90-102.

FESTO, AG \& Co. 2001. Fluidic muscle MAS. Accessed July 5. URL: http://www.festo.com/

Gavrilovic MM, Maric MR. 1969. Positional servo-mechanism activated by artificial muscles. Med Biol Eng, 7, 77-82.

Hill AV. 1996. The heat of shorting and the dynamic constants of muscle. In Proc R Soc, B, 126, p. 136-95.

Hildebrandt A, Sawodny O, Neumann R, et al. 2002. A Flatness based design for tracking control of pneumatic muscle actuators. In 7th International Conference on Control, Automation Robotics and Vision, Singapore, p. 1156-61.

Kawasaki H, Shimomura H, Shimizu Y. 2001.

Educational-industrial complex development of an anthropomorphic robot hand 'Gifu hand'. VSP and Robotics Society of Japan 2001. Adv Rob, 15(3), 357-63.

Klute GK, Czernieki JM, Hnnaford B. 1999. McKibben artificial muscles: pneumatic actuators with biomechanical intelligence. In Proceedings of the IEEE/ASME International Conference on Advanced Intelligent Mechatronics, Atlanta, GA, p. 221-6.

Liu H, Meusel P, Butterfass J, et al. 1998. DLR's multi sensory articulated hand Part I: hard- and software architecture. In IEEE Conference on Robotics and Automation, Leuven, Belgium, 16-20 May, p. 2087-93.

Nickel VL, Perry, Garrett AL. 1963. Development of useful function in the severely paralysed hand. 7 Bone foint Surg, $45 \mathrm{~A}(5), 933-52$. 
Noritsugu T, Kubota M, Yoshimatsu S. 2001. Development of pneumatic rotary soft actuator made of silicone rubber. 7 Rob Mechatron, 13-1, 17-22.

Repperger DW, Johnson KR, Philips CA. 1999. Nonlinear feedback controller design of a pneumatic muscle actuator system. In Proceedings of American Control Conference, San Diego, CA, p. 1525-9.

Saga N, Nakamura T, Uehara J, et al. 2002. Development of artificial muscle actuator reinforced by Kevlar fiber. In
Proceedings of IEEE International Conference on Industrial Technology, Bangkok, Thailand, p.950-4.

Saga N, Nakamura T, Ueda S. 2003. Study on peristaltic crawling robot using artificial muscle actuator. In IEEE/ASME International Conference on Advanced Intelligence Mechatronics, Kobe, Japan, p. 679-84

Yanagisawa T, Yagi S, Ide T, et al. 2001. Application of Pneumatic Silicone Rubber Pipe Actuator to Robotics. Research reports of the Kogakuin University, 91, p. 17-24. 

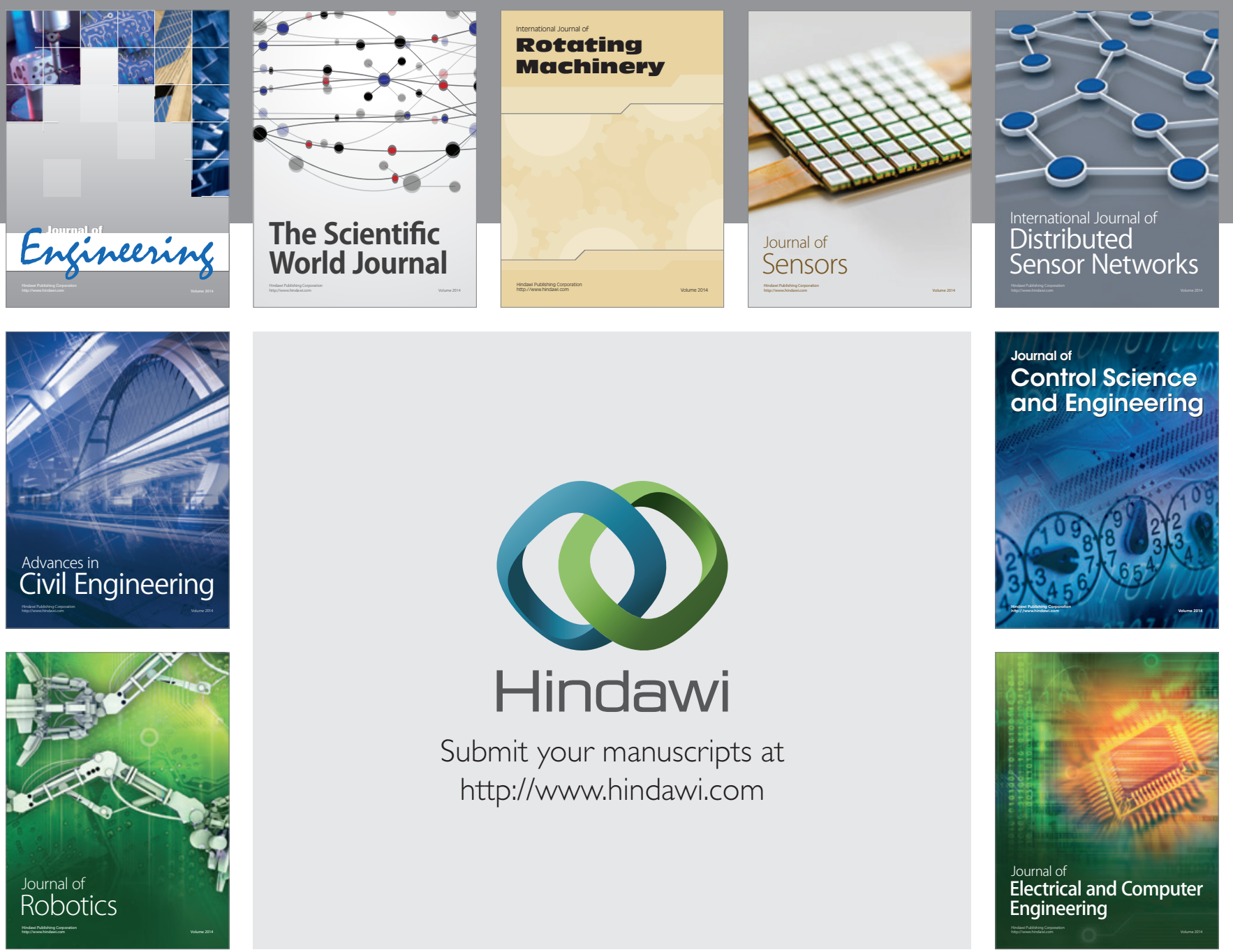

Submit your manuscripts at

http://www.hindawi.com
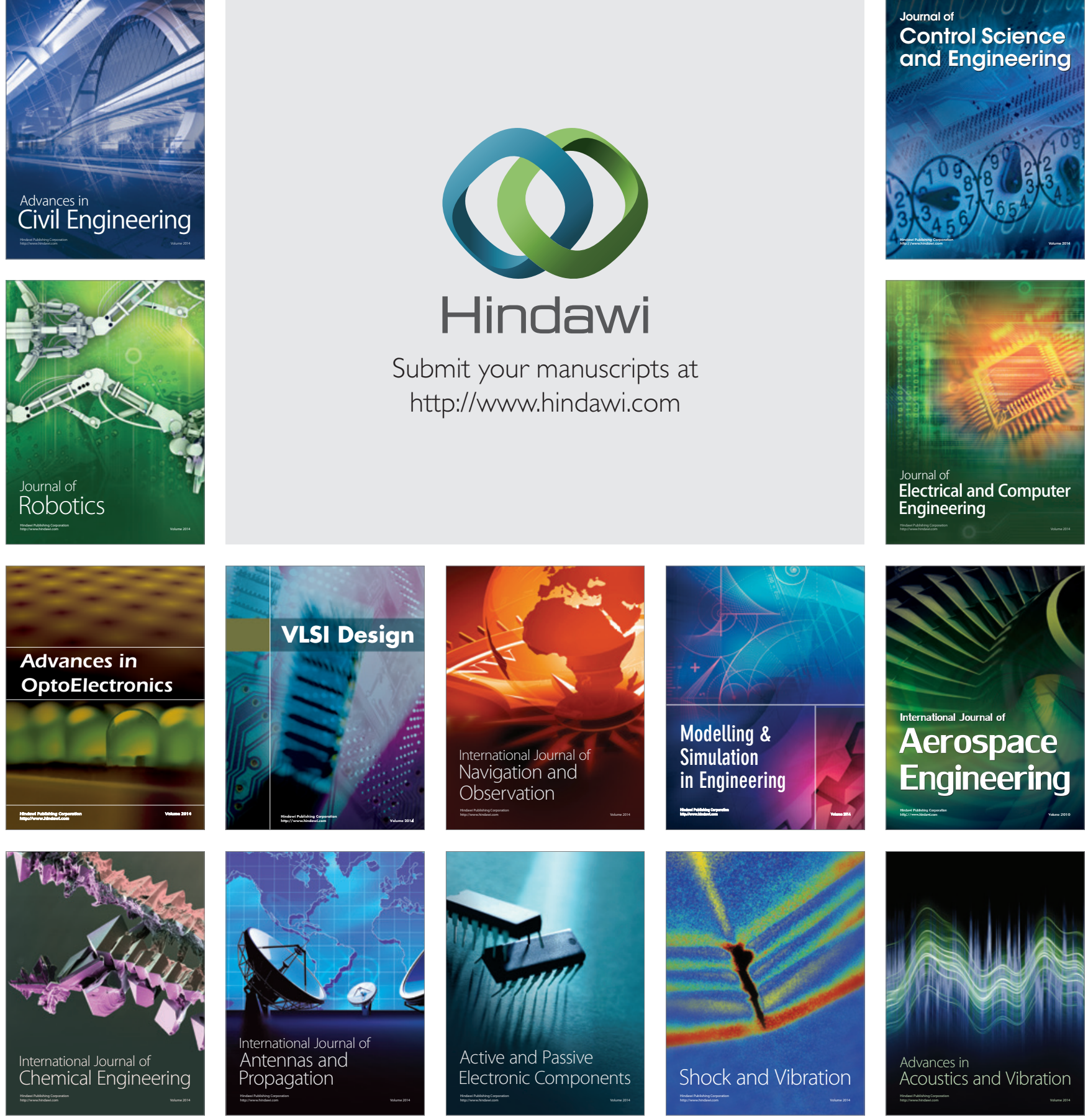